\title{
Application of NRS2002 in Preoperative Nutritional Screening for Patients with Liver Cancer
}

\author{
Suling Huang, ${ }^{1}$ Shijie Wang, ${ }^{1}$ Yuankang Xie, ${ }^{1}$ Xiao He, ${ }^{1}$ Xiuying Yi, ${ }^{1}$ Jianhong Zhang, \\ Zuomei Deng, ${ }^{3}$ and Ling Yin $\mathbb{D}^{1}$ \\ ${ }^{1}$ Department of Hepatological Surgery, First Affiliated Hospital of Gannan Medical University, Ganzhou 341000, \\ Jiangxi Province, China \\ ${ }^{2}$ Department of Pharmacy, First Affiliated Hospital of Gannan Medical University, Ganzhou 341000, Jiangxi Province, China \\ ${ }^{3}$ Department of Thyroid Hernia Surgery, First Affiliated Hospital of Gannan Medical University, Ganzhou 341000, \\ Jiangxi Province, China \\ Correspondence should be addressed to Ling Yin; yinling20210701@163.com
}

Received 1 July 2021; Accepted 17 August 2021; Published 27 August 2021

Academic Editor: Muhammad Wasim Khan

Copyright (c) 2021 Suling Huang et al. This is an open access article distributed under the Creative Commons Attribution License, which permits unrestricted use, distribution, and reproduction in any medium, provided the original work is properly cited.

Objective. To explore the application of NRS2002 in preoperative nutritional screening of patients with liver cancer (LC). Methods. 60 LC patients treated in the First Affiliated Hospital of Gannan Medical University (January 2018-May 2021) were chosen as the research objects, and split into group J without nutritional risk and group Q with nutritional risk according to the results of NRS2002 to compare the preoperative situation, surgery-related indexes, hematological indexes, postoperative recovery, and incidence of complications between the two groups. Results. Group J $(n=28)$ and group Q $(n=32)$ showed no obvious difference in preoperative situation, and patients' liver function indexes were within the normal range. The duration of surgery in group J was notably shorter compared with group Q $(P<0.05)$. Alanine aminotransferase $($ ALT), aspartate aminotransferase $($ AST $)$, direct bilirubin (DBIL), and albumin in group J were notably different from those of group Q $(P<0.001)$ at 1 day after surgery. ALT and AST in group J were notably different from those of group Q at 3 days after surgery $(P<0.001)$. No obvious differences were observed in the hematological indexes between the two groups at 5 days after surgery $(P>0.05)$. The total amount of albumin infusion, postoperative hospitalization time, and hospitalization cost in group J were notably lower compared with group Q $(P<0.001)$. The incidence of complications in group J was notably lower compared with group Q $(P<0.05)$. Conclusion. Postoperative recovery of LC patients is closely related to their preoperative nutritional status, and those with poor nutritional status have a high incidence of postoperative complications and long recovery time. NRS2002 can effectively screen the nutritional status of patients and provide reference for prognosis evaluation.

\section{Introduction}

Liver cancer (LC) is a common clinical disease. According to the data of International Agency for Research on Cancer (IARC), the number of LC cases in China accounts for 55\% of the total cases worldwide, and about $70 \%$ of the patients are in the middle and late stages when diagnosed, with an increasing annual mortality rate, endangering the life and health of Chinese residents. At present, surgical treatment is generally performed in practice to radically treat it. However, LC patients are often complicated with other diseases such as liver cirrhosis, their liver cells are seriously damaged, their metabolic function is reduced, and the inactivated function of steroid hormones is weakened, which jointly lead to the nutritional and metabolic imbalances in patients. Therefore, the possibility of malnutrition in patients with liver cancer during perioperative period reaches $80 \%$, and severe malnutrition will increase the probability of surgical complications, enhance the adverse reactions of radiotherapy and chemotherapy, and affect the prognosis of patients [1-3]. In recent years, precise requirements have been put forward for surgical procedures. Preoperative assessment 
has become an integral part of surgical procedures, including liver function classification and imaging examination in LC patients. However, there were few preoperative examinations related to nutritional assessment. Since clinical practice shows that there is a close relationship between the nutritional status of patients and their postoperative recovery, it is extremely important to add preoperative nutritional assessment to the scope of preoperative examination [4-6]. NRS2002 is a common nutritional risk-screening scale in clinical practice, which can be applied in various types of hospitalized patients. Based on this, this paper aims to explore the application of NRS2002 in preoperative nutritional screening of LC patients, summarized as follows.

\section{Materials and Methods}

2.1. Preoperative Assessment. Sixty LC patients treated in the First Affiliated Hospital of Gannan Medical University (January 2018-May 2021) were chosen as the research objects and underwent preoperative evaluation, with steps as follows. (1) All the patients received routine examination. (2) The patients received indocyanine green excretive test with 15-minute retention and other methods to evaluate their liver function $[7,8]$. (3) The patients underwent imaging examinations, including MRI examination and ultrasound examination to observe the tumor size, location, and other information and to determine whether extrahepatic metastasis occurred.

This study was in line with the principles of Declaration of Helsinki and was approved by the Ethics Committee of the First Affiliated Hospital of Gannan Medical University.

2.2. Inclusion Criteria. (1) The patients or their families fully recognized the study process and signed the informed consent. (2) The patients were diagnosed with LC after examination, and the lesions could be removed by hepatectomy. (3) The liver function of patients was graded as A $[9,10]$. (4) The 15-minute retention rate of indocyanine green excretion test was $20 \%$ and below.

2.3. Exclusion Criteria. (1) The patients had mental problems or could not communicate with others. (2) The patients had other organic diseases. (3) The patients had liver function grade below A. (4) The patients had extrahepatic metastasis of cancer or tumor thrombus in the portal vein and primary branches. (5) The patients received radiotherapy and chemotherapy before surgery. (6) The patients quit the study halfway.

\subsection{Methods}

2.4.1. Surgical Methods. (1) All surgeries of the patients were operated by the same surgical group. The surgical plans were selected according to the actual situation of the patients, and the ultrasound-assisted technology was adopted to clarify the sites for resection. (2) Portal triad clamping (Pringle) was selected for hepatic inflow occlusion. The liver tissue was separated by the fine clamp method, and the pipeline structure of the section was sutured, except for the liver section.

2.4.2. Perioperative Nutrition Support Schemes. (1) The patient did not take nutritional support before surgery and continued to follow the routine dietary. (2) After surgery, enteral nutrition support was given to the patients. The patients could drink water at $12 \mathrm{~h}$ after surgery. With low amount and high frequency, the patients drank enteral nutrient solution (Nutricia Pharmaceutical Co., Ltd., Wuxi Branch; NMPA approval no. H20030012) at $24 \mathrm{~h}$ after surgery with $500 \mathrm{ml}$ each day and was increased to $1000 \mathrm{~mL}$ within 3 days, accompanied by semiliquid diet.

\subsection{Observation Criteria}

(1) Grouping of patients: the patients were split into group J without nutritional risk (NRS2002 score <3 points) and group Q with nutritional risk (NRS2002 score $\geq 3$ points) according to the results of NRS2002, and the number of each group was counted [11-14].

(2) Preoperative situation: the comparison items included gender, age, underlying diseases, alanine aminotransferase (ALT) level, aspartate aminotransferase (AST) level, total bilirubin (TBIL) level, direct bilirubin (DBIL) level, and albumin level.

(3) Surgery-related indexes: the range of liver resection, duration of surgery, hepatic portal occlusion, intraoperative blood loss, and intraoperative blood transfusion were compared.

(4) Hematological indexes: the ALT, AST, TBIL, DBIL, and albumin levels [15-18] at 1 day, 3 days, and 5 days after surgery were compared.

(5) Postoperative recovery: the total amount of albumin infusion, postoperative ventilation time, postoperative hospitalization time, and hospitalization cost were compared.

(6) Incidence of complications: complications included ascites, bile leakage, abdominal infection, and postoperative hemorrhage. The number of patients with complications was counted.

2.6. Statistical Treatment. In this study, the data were processed by SPSS 20.0 and graphed by GraphPad Prism 7 (GraphPad Software, San Diego, USA). This study included enumeration data and measurement data, tested by $X^{2}$ and $t$-test. The difference was statistically significant when $P<0.05$.

\section{Results}

3.1. Analysis of Patient Grouping. The grouping of patients is shown in Figure 1.

3.2. Comparison of Preoperative Situation. No obvious difference in preoperative situation was found between the two 


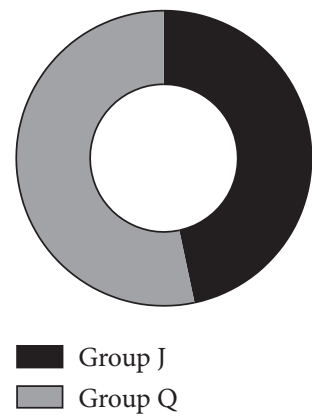

Figure 1: Analysis of patient grouping $(n(\%))$. The black area represents group J $(n=28,46.7 \%)$, and the gray area represents group Q $(n=32,53 \cdot 3 \%)$.

groups $(P>0.05)$, and the liver function indexes of all patients were within the normal range (Table 1$)$.

3.3. Comparison of Surgery-Related Indexes. The duration of surgery in group J was notably shorter compared with group $\mathrm{Q}(P<0.05$; Figure 2 and Table 2$)$.

Group J included 14 patients (50\%) with a resection of 3 segments or less and 14 patients (50\%) with a resection of more than 3 segments. Group Q included 12 patients $(37.5 \%)$ with a resection of 3 segments or less and 20 patients $(62.5 \%)$ with a resection of more than 3 segments. The comparison between the two groups showed $X^{2}=0.950$ and $P=0.330$.

3.4. Comparison of Hematological Indexes. ALT, AST, DBIL, and albumin in group J were notably different from those of group $Q(P<0.001)$ at 1 day after surgery. ALT and AST in group J were notably different from those of group $Q$ at 3 days after surgery $(P<0.001)$. No obvious differences were observed in the hematological indexes between the two groups at 5 days after surgery $(P>0.005)$ (see Table 3 ).

3.5. Comparison of Postoperative Recovery. The total amount of albumin infusion, postoperative hospitalization time, and hospitalization cost in group J were notably lower compared with group $\mathrm{Q}(P<0.001$; Table 4$)$.

3.6. Comparison of the Incidence of Complications. The incidence of complications in group $\mathrm{J}$ was notably lower compared with group $\mathrm{Q}(P<0.05$; Figure 3$)$.

\section{Discussion}

With the continuous progress of relevant medical technology in recent years and significantly improved level of liver cancer surgery in China, how to optimize the prognosis of LC patients undergoing surgery by preoperative assessment has become the focus of clinical research. Complication with underlying liver diseases such as liver cirrhosis in LC patients in China can increase the damage to liver cells and affect liver nutritional and metabolic functions. At the same time, due to the decline of appetite in LC patients and
TABLE 1: Comparison of preoperative situation.

\begin{tabular}{lcccc}
\hline Group & $\begin{array}{c}\text { Group J } \\
(n=28)\end{array}$ & $\begin{array}{c}\text { Group Q } \\
(n=32)\end{array}$ & $X^{2} / t$ & $P$ \\
\hline Gender & & & 0.234 & 0.628 \\
$\quad$ Male & $14(50.0 \%)$ & $14(43.8 \%)$ & & \\
Female & $14(50.0 \%)$ & $18(56.3 \%)$ & & \\
\hline Age (years old) & & & & \\
$\quad$ Range & $32-74$ & $33-74$ & & \\
Average age & $51.21 \pm 6.20$ & $51.23 \pm 6.21$ & 0.012 & 0.990 \\
\hline Underlying diseases & & & & \\
Diabetes & $6(21.4 \%)$ & $10(31.3 \%)$ & 0.737 & 0.391 \\
Hypertension & $3(10.7 \%)$ & $6(18.8 \%)$ & 0.756 & 0.384 \\
ALT (U/L) & $38.98 \pm 9.12$ & $42.11 \pm 9.65$ & 1.286 & 0.204 \\
AST (U/L) & $30.15 \pm 4.65$ & $32.15 \pm 5.36$ & 1.533 & 0.131 \\
TBIL $(\mu \mathrm{mol} / \mathrm{L})$ & $14.99 \pm 0.54$ & $15.10 \pm 0.65$ & 0.707 & 0.482 \\
DBIL $(\mu \mathrm{mol} / \mathrm{L})$ & $4.10 \pm 0.35$ & $4.24 \pm 0.65$ & 1.017 & 0.313 \\
Albumin $(\mathrm{g} / \mathrm{L})$ & $40.10 \pm 0.68$ & $39.98 \pm 1.54$ & 0.381 & 0.705 \\
\hline
\end{tabular}

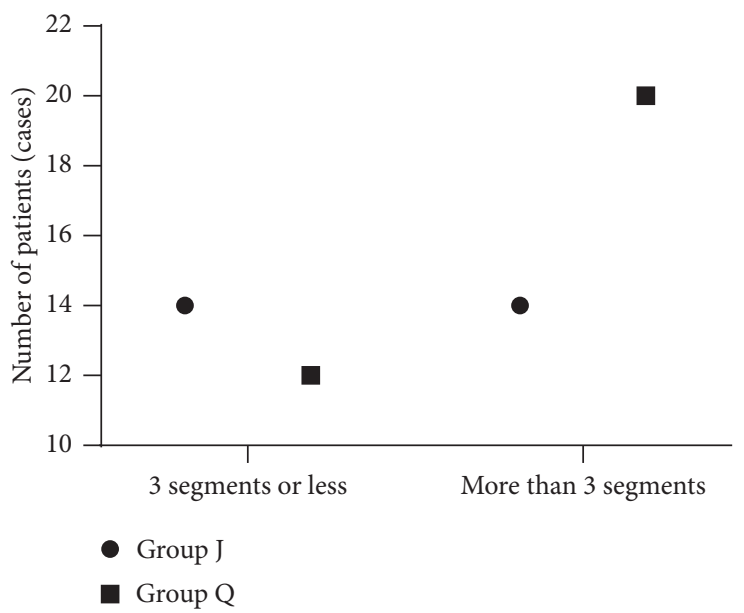

Figure 2: Scope of liver resection. The abscissa from left to right represent 3 segments or less and more than 3 segments, respectively, and the ordinate represents the number of patients (cases). The dots represent group J, and the squares represent group Q.

reduced nutrition intake with different degrees of Warburg effect, the incidence of malnutrition in patients can reach $80 \%$, adversely affecting the postoperative recovery of patients [19-21]. At present, the preoperative assessment of liver cancer patients includes liver function classification and hepatic functional reserve test, while nutritional assessment is not included. However, clinical practice has confirmed the key role of nutritional assessment, so close attention should be paid to the selection of appropriate preoperative nutritional assessment tools in doctors.

As a common preoperative nutritional assessment tool in clinical practice, NRS2002 is superior to other assessment scales in sensitivity, which can be adopted to screen nutritional risk indicators of hospitalized patients and is associated with the prognosis of patients [22, 23]. NRS2002 can be used in the preoperative assessment of various diseases. In this study, it was applied to the preoperative nutritional assessment of LC patients, and 60 patients were split into group J $(n=28)$ without nutritional risk and 
TABle 2: Comparison of surgery-related indexes.

\begin{tabular}{lccr}
\hline Items & $\begin{array}{c}\text { Group J } \\
(n=28)\end{array}$ & $\begin{array}{c}\text { Group Q } \\
(n=32)\end{array}$ & $X^{2} / t$ \\
\hline Duration of surgery (min) & $215.68 \pm 26.87$ & $365.98 \pm 35.15$ & 18.399 \\
\hline $\begin{array}{l}\text { Hepatic portal occlusion } \\
\quad \text { Yes }\end{array}$ & $14(50.0 \%)$ & $20(62.5 \%)$ & 0.950 \\
$\quad$ No & $14(50.0 \%)$ & $12(37.5 \%)$ & 0.330 \\
\hline Intraoperative blood loss (ml) & $295.12 \pm 60.98$ & $565.98 \pm 48.45$ & 19.156 \\
\hline Intraoperative blood transfusion & $4(14.3 \%)$ & $10(31.3 \%)$ & 2.402 \\
$\quad$ Yes & $24(85.7 \%)$ & $22(68.8 \%)$ & 0.121 \\
$\quad$ No & & \\
\hline
\end{tabular}

TABLE 3: Comparison of hematological indexes $(\bar{x} \pm s)$.

\begin{tabular}{|c|c|c|c|c|}
\hline Items & $\begin{array}{l}\text { Group J } \\
(n=28)\end{array}$ & $\begin{array}{c}\text { Group Q } \\
(n=32)\end{array}$ & $t$ & $P$ \\
\hline \multicolumn{5}{|l|}{1 day after surgery } \\
\hline ALT (U/L) & $205.65 \pm 28.54$ & $489.35 \pm 65.12$ & 21.314 & $<0.001$ \\
\hline AST (U/L) & $180.54 \pm 25.48$ & $470.65 \pm 98.54$ & 15.128 & $<0.001$ \\
\hline TBIL $(\mu \mathrm{mol} / \mathrm{L})$ & $20.99 \pm 2.54$ & $22.65 \pm 5.21$ & 1.533 & 0.131 \\
\hline DBIL $(\mu \mathrm{mol} / \mathrm{L})$ & $6.10 \pm 0.54$ & $13.54 \pm 3.68$ & 10.588 & $<0.001$ \\
\hline Albumin $(\mathrm{g} / \mathrm{L})$ & $32.11 \pm 0.65$ & $30.98 \pm 1.20$ & 4.442 & $<0.001$ \\
\hline \multicolumn{5}{|l|}{3 days after surgery } \\
\hline $\operatorname{ALT}(\mathrm{U} / \mathrm{L})$ & $178.65 \pm 24.65$ & $356.98 \pm 98.52$ & 9.317 & $<0.001$ \\
\hline AST (U/L) & $75.45 \pm 10.58$ & $210.65 \pm 92.12$ & 7.714 & $<0.001$ \\
\hline TBIL $(\mu \mathrm{mol} / \mathrm{L})$ & $22.65 \pm 2.54$ & $23.65 \pm 2.14$ & 1.655 & 0.103 \\
\hline DBIL $(\mu \mathrm{mol} / \mathrm{L})$ & $8.21 \pm 1.68$ & $9.11 \pm 2.15$ & 1.788 & 0.079 \\
\hline Albumin $(\mathrm{g} / \mathrm{L})$ & $34.12 \pm 1.65$ & $33.68 \pm 0.65$ & 1.391 & 0.169 \\
\hline \multicolumn{5}{|c|}{5 days after surgery } \\
\hline $\operatorname{ALT}(\mathrm{U} / \mathrm{L})$ & $120.65 \pm 25.65$ & $125.98 \pm 26.98$ & 0.781 & 0.438 \\
\hline AST $(\mathrm{U} / \mathrm{L})$ & $46.98 \pm 4.25$ & $49.11 \pm 5.87$ & 1.589 & 0.117 \\
\hline TBIL $(\mu \mathrm{mol} / \mathrm{L})$ & $20.12 \pm 2.35$ & $20.98 \pm 2.68$ & 1.313 & 0.195 \\
\hline DBIL $(\mu \mathrm{mol} / \mathrm{L})$ & $7.10 \pm 1.24$ & $7.65 \pm 1.64$ & 1.448 & 0.153 \\
\hline Albumin $(\mathrm{g} / \mathrm{L})$ & $35.98 \pm 0.64$ & $35.87 \pm 0.98$ & 0.507 & 0.614 \\
\hline
\end{tabular}

TABLE 4: Comparison of postoperative recovery $(\bar{x} \pm s)$.

\begin{tabular}{|c|c|c|c|c|}
\hline Items & $\begin{array}{l}\text { Group J } \\
(n=28)\end{array}$ & $\begin{array}{c}\text { Group Q } \\
(n=32)\end{array}$ & $t$ & $P$ \\
\hline Total amount of albumin infusion $(\mathrm{g})$ & $13.10 \pm 3.48$ & $41.65 \pm 13.57$ & 10.815 & $<0.001$ \\
\hline Postoperative ventilation time (d) & $2.56 \pm 0.35$ & $2.68 \pm 0.35$ & 1.325 & 0.190 \\
\hline Postoperative & $9.25 \pm 0.30$ & $13.11 \pm 0.58$ & 31.680 & $<0.001$ \\
\hline
\end{tabular}

group Q $(n=32)$ with nutritional risk according to the classification of NRS2002. The two groups showed no obvious difference in preoperative situation, and patients' liver function indexes were within the normal range, which could be used for study.

Anesthesia, trauma, and other factors during liver cancer surgery can cause high catabolism. If patients have poor preoperative nutritional status and low surgical tolerance, their perioperative body consumption will be higher than that of ordinary patients, further increasing the possibility of metabolic disorders after surgery and seriously affecting the recovery during anesthesia. Moreover, liver cancer surgery will lead to serious damage to liver function. Malnutrition will further aggravate liver damage, significantly reduce the frequency of albumin synthesis, and increase the incidence of complications such as hypoproteinemia, infection, and poor healing, thus slowing the recovery of patients after surgery. This study showed that the duration of surgery and postoperative hospitalization time in group J were notably shorter compared with group $\mathrm{Q}(P<0.05)$, indicating that malnutrition will worsen the body condition of patients and increase the surgical risk. ALT, AST, DBIL, and albumin in group J were notably different compared with group Q $(P<0.001)$ at 1 day after surgery. ALT and AST in group $J$ were notably different compared with group $Q$ at 3 days after surgery $(P<0.001)$. The above results indicated that patients with malnutrition had more serious liver damage, with unsatisfactory postoperative recovery. 


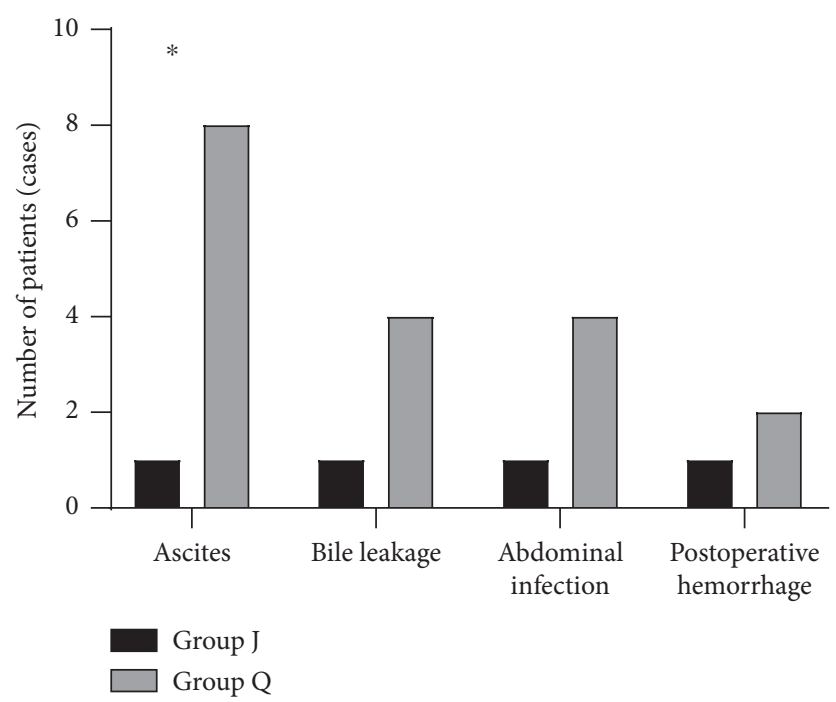

Figure 3: Comparison of the incidence of complications. The abscissa from left to right represent ascites, bile leakage, abdominal infection, and postoperative hemorrhage, and the ordinate represents the number of patients (cases). The black area represents group J, and the gray area represents group. Q ${ }^{*} P<0.05$. Ascites occurred in 1 case of group J and 8 cases of group Q. Bile leakage occurred in 1 case of group J and 4 cases of group Q. Abdominal infection occurred in 1 case of group J and 4 cases of group Q. Postoperative hemorrhage occurred in 1 case of group J and 2 cases of group Q.

The study also found that the hospitalization cost in group J was notably lower compared with group Q $(P<0.001)$, which may be related to the higher incidence of complications in group Q. Group Q received more albumin infusion, resulting in an obvious increase of the hospitalization cost. This study also showed that patients with malnutrition were more likely to have complications such as ascites and bile leakage compared with group J, but no serious complications or death occurred. This is because the patients in this study received comprehensive evaluation and perioperative management before surgery, as well as enteral nutrition support after surgery. Therefore, both groups of patients had certain recovery.

According to the study of Debanjan et al., the albumin infusion volume of patients with malnutrition undergoing liver cancer surgery was $(41.65 \pm 13.57) \mathrm{g}$, which was significantly higher than that of patients with normal nutrition $(P<0.001)$ [24], revealing that malnutrition can affect the body state and aggravate the perioperative consumption of patients. Therefore, it is of great importance to deepen the study on chronic nutritional consumption of such patients.

In conclusion, liver cancer is a common malignant disease affecting life and health of Chinese residents. This study has confirmed that postoperative recovery of LC patients is closely related to their preoperative nutritional status, and those with poor nutritional status have a high incidence of postoperative complications and long recovery time. NRS2002 can effectively screen the nutritional status of patients and provide reference for prognosis evaluation and the application of nutritional support programs.

\section{Data Availability}

All data can be provided by the corresponding author upon request.

\section{Conflicts of Interest}

The authors declare that they have no conflicts of interest.

\section{Authors' Contributions}

Suling Huang and Shijie Wang contributed equally to this work.

\section{Acknowledgments}

This article was funded by "Ganzhou Guiding Science and Technology Plan Task (GZ2018ZSF088).”

\section{References}

[1] R. Boulhosa, R. P. Loureno, D. M. Crtes et al., "Comparison between criteria for diagnosing malnutrition in patients with advanced chronic liver disease: GLIM group proposal versus different nutritional screening tools," Journal of Human Nutrition and Dietetics, vol. 33, no. 6, 2020.

[2] G. Giorgetti, F. Fabiocchi, G. Brandimarte, and A. Tursi, "Acute diverticulitis is at significant risk of malnutrition: an analysis of hospitalized patients in a medicine department," Journal of gastrointestinal and liver diseases: JGLD, vol. 28, no. 4, pp. 53-56, 2019.

[3] A. Adiamah, P. Skorepa, A. Weimann et al., "The impact of preoperative immune modulating nutrition on outcomes in patients undergoing surgery for gastrointestinal cancer: a systematic review and meta-analysis," Annals of Surgery, vol. 270, no. 2, pp. 247-256, 2019.

[4] K. Russell, H. G. Zhang, L. K. Gillanders et al., "Preoperative immunonutrition in patients undergoing liver resection: a prospective randomized trial," World Journal of Hepatology, vol. 11, no. 3, pp. 305-317, 2019.

[5] I. Bachini, V. Ariu, F. Canaletti et al., "IMMUNO-ERAS: preoperative immunonutrition IN patients undergoing 
elective liver surgery within eras pathway," Nutrition, vol. 7576, Article ID 110926, 2020.

[6] A. Demagistris, V. Ariu, I. Bachini et al., "Preoperative immunonutrition in liver surgery within eras pathway: a post-hoc analysis in the subgroup of patients at nutritional risk," Nutrition, vol. 75-76, Article ID 110918, 2020.

[7] P. Probst, J. Fuchs, M. R. Schön et al., "Prospective study to evaluate the prognostic value of different nutritional assessment scores in liver surgery: NURIMAS Liver (DRKS00006340)," Hepatobiliary Surgery and Nutrition, vol. 9, no. 4, pp. 400-413, 2020.

[8] P. Achilli, M. Mazzola, C. L. Bertoglio et al., "Preoperative immunonutrition in frail patients with colorectal cancer: an intervention to improve postoperative outcomes," International Journal of Colorectal Disease, vol. 35, no. 9868, 2020.

[9] R. Tokunaga, S. Nakagawa, Y. Miyamoto et al., "The clinical impact of preoperative body composition differs between male and female colorectal cancer patients," Colorectal Disease, vol. 22, no. 1, pp. 62-70, 2020.

[10] L. A. Robinson, T. Tanvetyanon, D. Grubbs et al., "Preoperative nutrition-enhanced recovery after surgery (N-ERAS) protocol for thoracic neoplasms," The Journal of Thoracic and Cardiovascular Surgery, vol. 162, no. 3, pp. 710.e1-720.e1, 2020.

[11] Y. Okugawa, "P2-080 clinical burden of prognostic nutrition index in patients with rectal cancer who receive preoperative chemoradiotherapy," Annals of Oncology, vol. 29, no. 7, 2018.

[12] N. Kamo, T. Kaido, Y. Miyachi et al., "Preoperative abnormal body composition is closely related to bacteremia after living donor liver transplantation," Nutrition, vol. 77, Article ID 110798, 2020.

[13] D. Atieh, M. Darbandi, A. Shariati et al., "The effects of probiotics on reducing the colorectal cancer surgery complications: a periodic review during 2007-2017," Clinical Nutrition, vol. 39, no. 8, pp. 2358-2367, 2019.

[14] Korean Liver Cancer Association and National Cancer Center, "2018 Korean liver cancer association-national cancer center korea practice guidelines for the management of hepatocellular carcinoma," Gut \& Liver, vol. 13, no. 3, pp. 227-299, 2019.

[15] E. B. Tapper and N. D. Parikh, "Mortality due to cirrhosis and liver cancer in the United States, 1999-2016: observational study," BMJ, vol. 362, p. k2817, 2018.

[16] J. L. Petrick, T. Jake, Z. J. Anne et al., "Body mass index, diabetes and intrahepatic cholangiocarcinoma risk: the liver cancer pooling project and meta-analysis," The American Journal of Gastroenterology, vol. 113, no. 10, pp. 1494-1505, 2018.

[17] P. C. Valery, M. Laversanne, P. J. Clark, J. L. Petrick, K. A. McGlynn, and F. Bray, "Projections of primary liver cancer to 2030 in 30 countries worldwide," Hepatology, vol. 67, pp. 600-611, 2018.

[18] A. Chatterjee, J. Biswas, A. Chatterjee, S. Bhattacharya, B. Mukhopadhyay, and S. Mandal, "Psorinum therapy in treating stomach, gall bladder, pancreatic, and liver cancers: a prospective clinical study," Evidence-Based Complementary and Alternative Medicine, vol. 2018, Article ID 724743, 7 pages, 2018

[19] A. Cast, L. Valanejad, M. Wright et al., "C/EBP $\alpha$-dependent preneoplastic tumor foci are the origin of hepatocellular carcinoma and aggressive pediatric liver cancer," Hepatology, vol. 67, no. 5, 2018.

[20] Y. Pang, C. Kartsonaki, I. Turnbull et al., "Diabetes, plasma glucose and incidence of fatty liver, cirrhosis and liver cancer: a prospective study of 0.5 million people," Hepatology, vol. 68 , 2018.

[21] A. W. Singer, K. R. Reddy, L. E. Telep et al., "Direct-acting antiviral treatment for hepatitis $\mathrm{C}$ virus infection and risk of incident liver cancer: a retrospective cohort study," Alimentary Pharmacology \& Therapeutics, vol. 47, no. 9, pp. 12781287, 2018.

[22] E. I. Cohen, D. Field, G. E. Lynskey, and A. Y. Kim, "Technology of irreversible electroporation and review of its clinical data on liver cancers," Expert Review of Medical Devices, vol. 15, no. 2, pp. 99-106, 2018.

[23] S. T. Orcutt and D. A. Anaya, "Liver resection and surgical strategies for management of primary liver cancer," Cancer Control: Journal of the Moffitt Cancer Center, vol. 25, no. 1, 2018.

[24] D. Debanjan, A. Laura, N. Hayato et al., "The application value of NRS2002 in patients with liver cancer, the relationship between nutritional status and patient prognosis," Cancer Cell, vol. 33, no. 6, pp. 1061.e6-1077.e6, 2018. 